\title{
ON STABLE CHAOS IN THE ASTEROID BELT
}

\author{
MILOŠ ŠIDLICHOVSKÝ \\ Astronomical Institute Prague, Boðní II 1401, 14131 Praha 4, Czech Republic
}

\begin{abstract}
The twenty most chaotic objects found among first hundred of numbered asteroids are studied. Lyapunov time calculated with and without inner planets indicates that for eleven of those asteroids the strongest chaotic effect results from the resonances with Mars. The filtered semimajor axis displays an abrupt variation only when a close approach to Mars takes place. The study of the behaviour of the critical argument for candidate resonances can reveal which is responsible for the semimajor axis variation. We have determined these resonances for the asteroids in question. For the asteroids chaotic even without the inner planets we have determined the most important resonances with Jupiter, or three-body resonances.
\end{abstract}

\section{Introduction}

The label "stable chaos" was introduced by Milani and Nobili (1992) for chaos in systems for which no macroscopic changes are observed over a time period longer than $1000 T_{L}$ ( $T_{L}$ is the Lyapunov time). Asteroid $522 \mathrm{Helga}$ (with $L_{T}=6900 \mathrm{yr}$ ) was the first clear-cut example of stable chaos. Many other asteroids have been found to have $T_{L}$ much shorter than their orbital stability, and stable chaos now seems to be a common phenomenon. Milani and Nobili (1992) showed that the strongest chaotic effects for Helga are caused by the interaction of the intermediate order resonance 12:7 with secular perturbations acting on the eccentricity and perihelion of the asteroid. Šidlichovský and Nesvorný (1998) applied their modification (Šidlichovský and Nesvorný, 1998) of Laskar method (Laskar, 1990) to the first one hundred of the numbered asteroids and, as a by-product, obtained the Lyapunov times for each studied asteroid. These numerical calculations were performed using Nesvorný's MSI package (Šidlichovský and Nesvorný, 1994) for the Sun, 7 planets (without Mercury and Neptune) and the asteroid.

The integrator was the 12th-order symmetric multistep method by Quinlan and Tremaine (1990). The integration step was one day. Table I shows the twenty most chaotic asteroids of the studied sample. Column 3 shows the Lyapunov time ( 7 planets used).

The degree of chaos may be studied using many other tools. Froeschlé et al. (1998) calculated the so-called Fast Lyapunov Indicators (Lega and Froeschlé, 1997) for 5400 asteroids without the inner planets. By comparing their list of 848 most chaotic asteroids with ours we found that all their chaotic asteroids (belonging to the first one hundred numbered asteroids) were rather chaotic in our calculation as well, but we also found many others. The most important difference in our calculations was that we took into account Mars, Venus and the Earth. This suggests that the additional chaos was caused by the inner planets, especially Mars.

Holman and Murray (1996) studied the variation of Lyapunov time as a function of the initial semimajor axis throughout the outer asteroid belt in the threedimensional, elliptic, restricted, three-body problem. They observed $T_{L}$ in the $a-T_{L}$ 


\section{TABLE I}

The twenty most chaotic asteroids ( $T_{L}>32000 \mathrm{yr}$ ) of the first one hundred numbered asteroids. Integrations are performed with 7 planets, Column 3 and 4 planets, Column 4. Column 5 shows the resonance with the strongest chaotic effect.

\begin{tabular}{rlrrl}
\hline No. & name & $\begin{array}{r}\bar{L}_{T}\left[10^{3} \text { yr }\right] \\
7 \text { planets }\end{array}$ & $\begin{array}{r}L_{T}\left[10^{3} \text { yr }\right] \\
\text { 4 planets }\end{array}$ & resonance \\
\hline 2 & Pallas & 10 & 23 & $18: 7$ with Jupiter \\
7 & Iris & 17 & $>1000$ & $25: 49$ with Mars \\
8 & Flora & 30 & $>1038$ & $19: 33$ with Mars \\
10 & Hygiea & 16 & 15 & $8-3-4$ Jupiter-asteroid-Saturn \\
12 & Victoria & 33 & $>994$ & $29: 55$ with Mars \\
15 & Eunomia & 25 & $>980$ & $7: 16$ with Mars \\
23 & Thalia & 25 & $>1233$ & \\
33 & Polyhymnia & 10 & 14 & $22: 9$ with Jupiter \\
35 & Leukothea & 20 & 17 & \\
36 & Atlante & 4 & 5 & $4-2-3$ Jupiter-asteroid-Saturn \\
41 & Daphne & 14 & $>360$ & $9: 22$ with Mars \\
46 & Hestia & 30 & $>1039$ & \\
50 & Virginia & 10 & 12 & $11: 4$ with Jupiter \\
53 & Kalypso & 19 & 14 & $6-2-1$ Jupiter-asteroid-Saturn \\
60 & Echo & 27 & $>1159$ & \\
70 & Panopea & 24 & 36 & $2-1-2$ Jupiter-asteroid-Saturn \\
75 & Eurydike & 16 & $>932$ & \\
78 & Diana & 13 & 149 & \\
79 & Eurynome & 32 & 497 & \\
86 & Semele & 6 & 6 & $13: 6$ with Jupiter
\end{tabular}

graph corresponding to mean motion resonances and verified that the resonant angle makes transitions between circulation in one sense to circulation in the other sense, interspaced with brief periods of librations. Morbidelli and Nesvorny (1998) made similar calculations for the inner belt including the planets (even Mars) and observed peaks in the a-maximum LCE graph (which are the same as the dips in the $a-T_{L}$ graph). They could identify many of these peaks with mean motion resonances, but there were many other peaks which were finally identified with three-body mean motion resonances. These resonances correspond to the relation between mean motions of Jupiter, asteroid and Saturn:

$$
m_{J} \dot{\lambda}_{J}+m \dot{\lambda}+m_{S} \dot{\lambda}_{S} \sim 0
$$

where $m, m_{J}$ and $m_{S}$ are integers. The analytical model of the three-body resonances was presented by Nesvorny and Morbidelli (1998). Once this relation of resonances to low $T_{L}$ has been established, we may ask which resonances are mainly responsible for the chaotic behaviour of the most chaotic large asteroids in Tab. 1. This is the question we try to answer and check out by studying the behaviour of the corresponding critical argument with the relation to the variation of the filtered semimajor axis. 

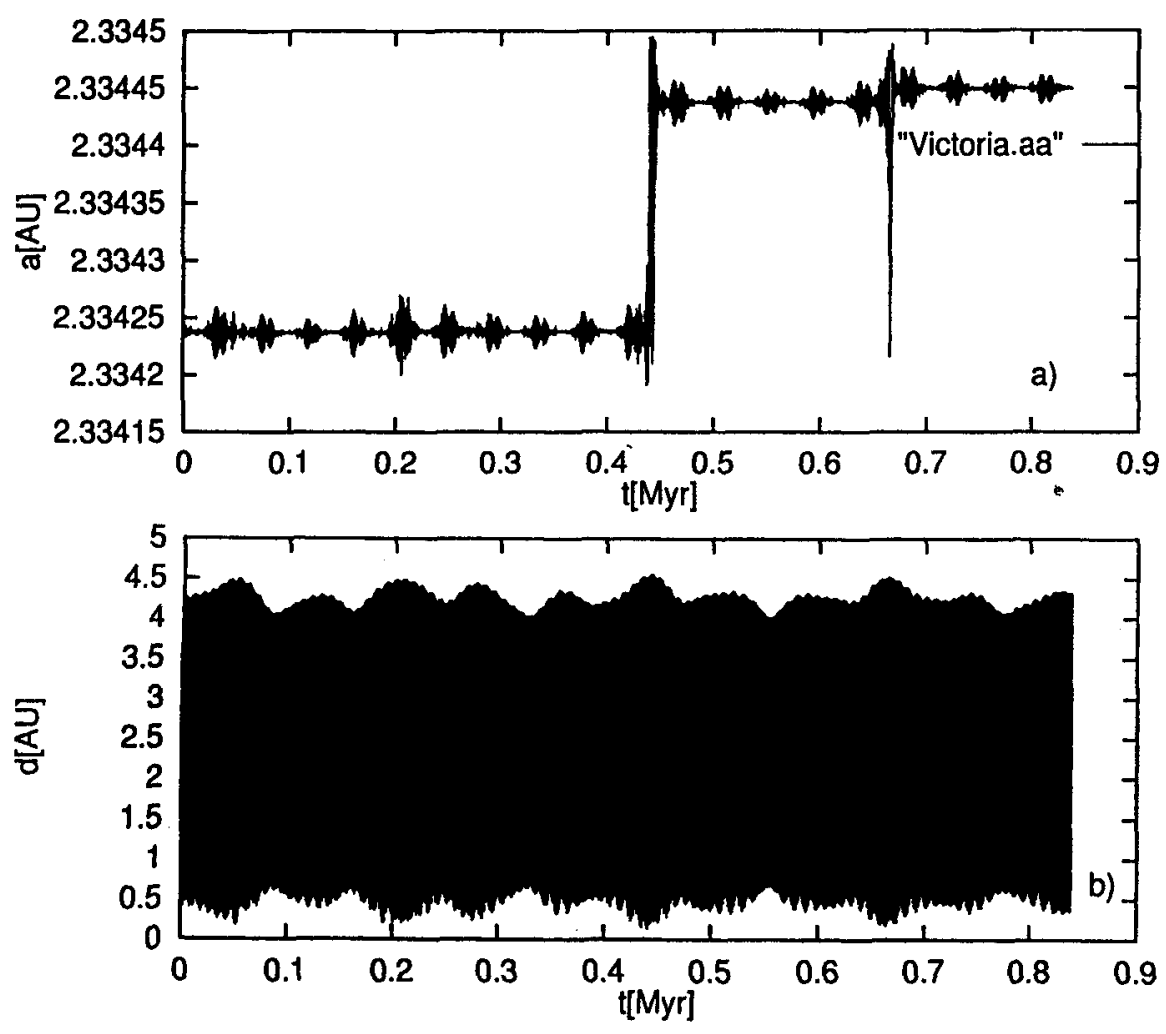

Fig. 1.

a)The filtered semimajor axis of Victoria b)The distance between Victoria and Mars

\section{Effect of Mars}

We calculated the Lyapunov time for the sample of twenty asteroids again, but now without the inner planets. The result is shown in Column 4 of Tab. 1. Our sample seems to split into two groups:

1) Asteroids with higher degree of chaoticity only when inner planets are considered: Iris, Flora, Victoria, Eunomia, Thalia, Daphne, Hestia, Echo, Eurydike, Diana, Eurynome

2) Asteroids with higher degree of chaoticity even without considering the inner planets: Pallas, Hygiea, Polyhymnia, Leukothea, Atlante, Virginia, Kalypso, Panopea, Semele

In studying the first group, we attempted to find some important resonances with Mars as it is the closest inner planet. We considered the digitally filtered output of the asteroid semi-major axis $a$ for about 800 thousand yrs. Filter A of Quinn et al. 

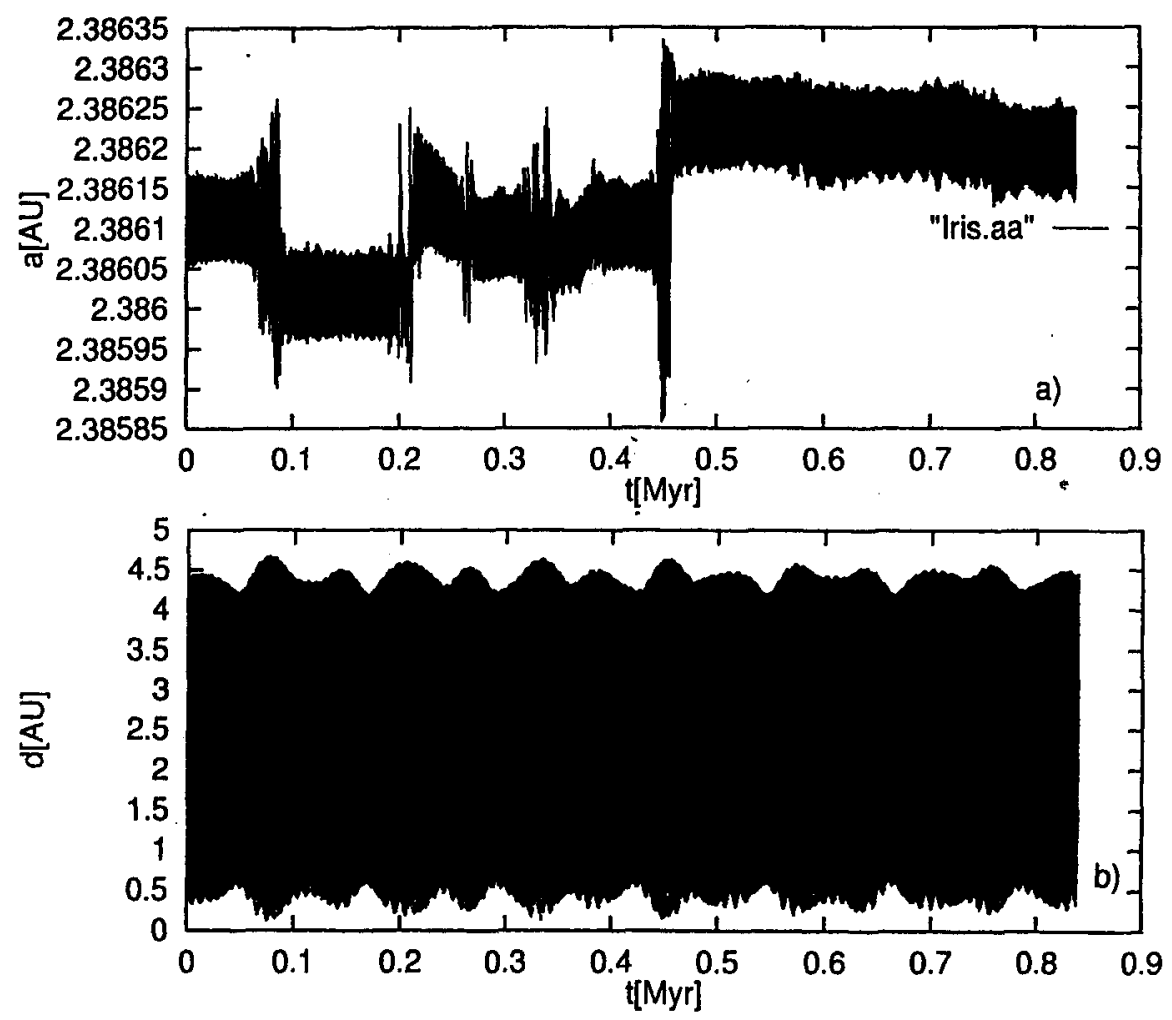

Fig. 2.

a) The filtered semimajor axis of Iris b) The distance between Iris and Mars

(1991) was used. As our output step in these calculations was 300 days, and filter A was applied twice, frequencies with periods shorter than 82 days were filtered out.

Semimajor axis $a$ could vary only very slowly after removing the short period terms, and, outside the resonance, it should be constant in the theories of second order in small parameter $\mu$ (Jupiter mass). Figure 1a shows filtered $a$ for asteroid Victoria. In this period two jumps in $a$ can be seen. The larger is about $0.0002 \mathrm{AU}$. The amplitudes of short-period variations in $a$ are of the order $0.005 \mathrm{AU}$, hence filtration is required. Figure $1 \mathrm{~b}$ shows the distance between Victoria and Mars. It is clear that the abrupt variation in $a$ is correlated to the short approach to Mars. It is not actually a close encounter as the asteroid stays well outside the Martian Hill sphere, but a temporary capture into the resonance with Mars is possible.

This is the common behaviour of the asteroids of the first group, except for Hestia and Eurynome. Figure 2 shows the same graphs for Iris. The jumps in $a$ 

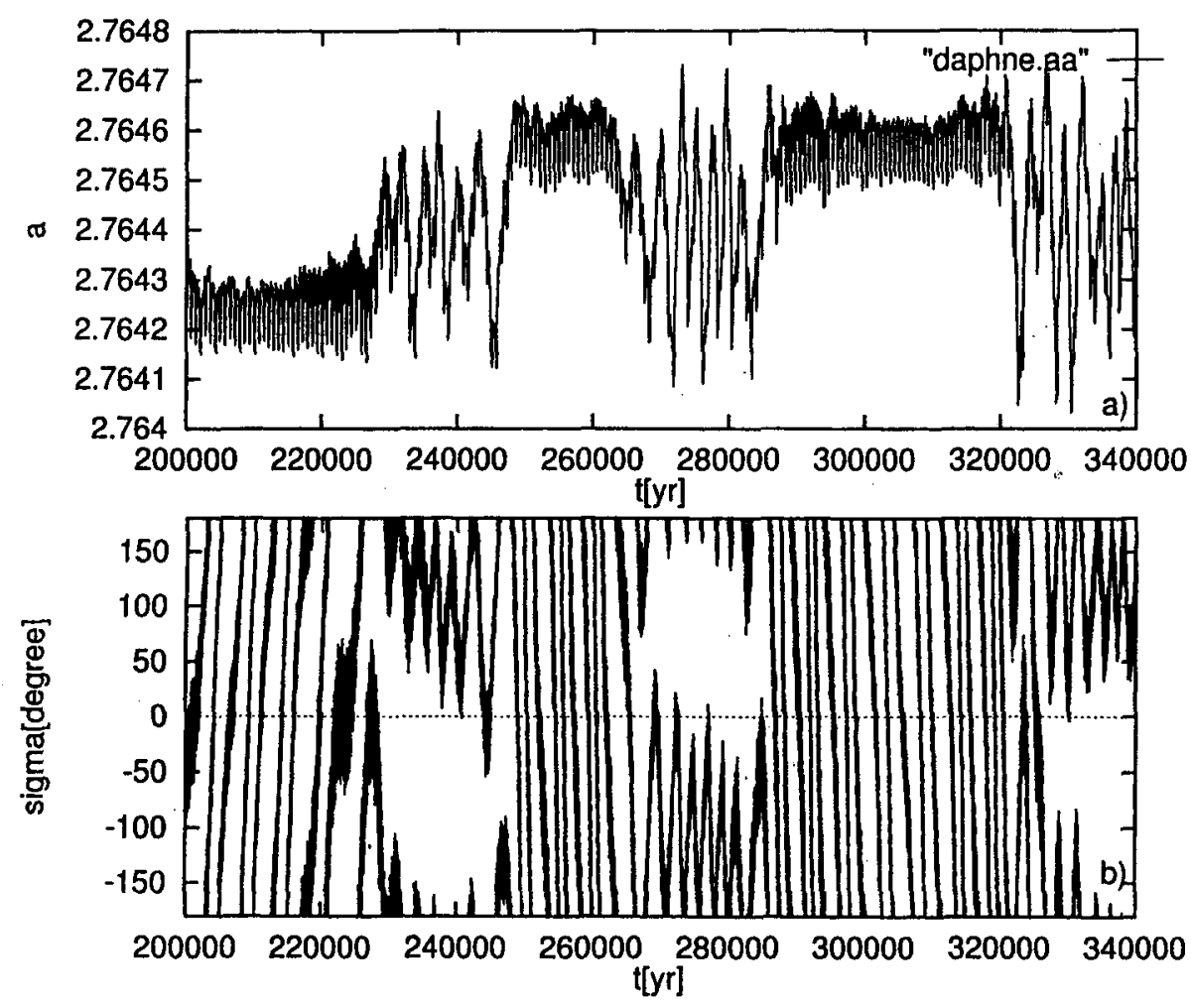

Fig. 3.

a) The filtered semimajor axis of Daphne b) The critical argument of Daphne: $\sigma=22 \lambda-9 \lambda_{M}-13 \varpi$

usually start as larger oscillations (presumably resonant) with transition to small amplitude oscillations around the new $a$ value.

As the next step we made extensive tables of position (value $a$ ) of the resonances with Mars to order 30. For the studied asteroid we can read $a$ from the graph and find the close candidates for the resonance from the tables. We can then check the behaviour of the corresponding critical arguments. Figure 3a shows filtered $a$ for Daphne. Three regions of the resonant $a$ oscillations can be identified. Fig. $3 \mathrm{~b}$ shows the corresponding resonant argument $\sigma=22 \lambda-9 \lambda_{M}-13 \varpi$. Here $\lambda$ stands for the mean longitude, $\varpi$ for the longitude of the perihelion, and subscript $M$ for Mars.

We see the transitions between circulation and librations with correlation to the $a$ variations. This is evidence that resonance $9 / 22$ with Mars is the most important for chaotic behaviour of Daphne. Figure 4 shows filtered $a$ and the critical argument 


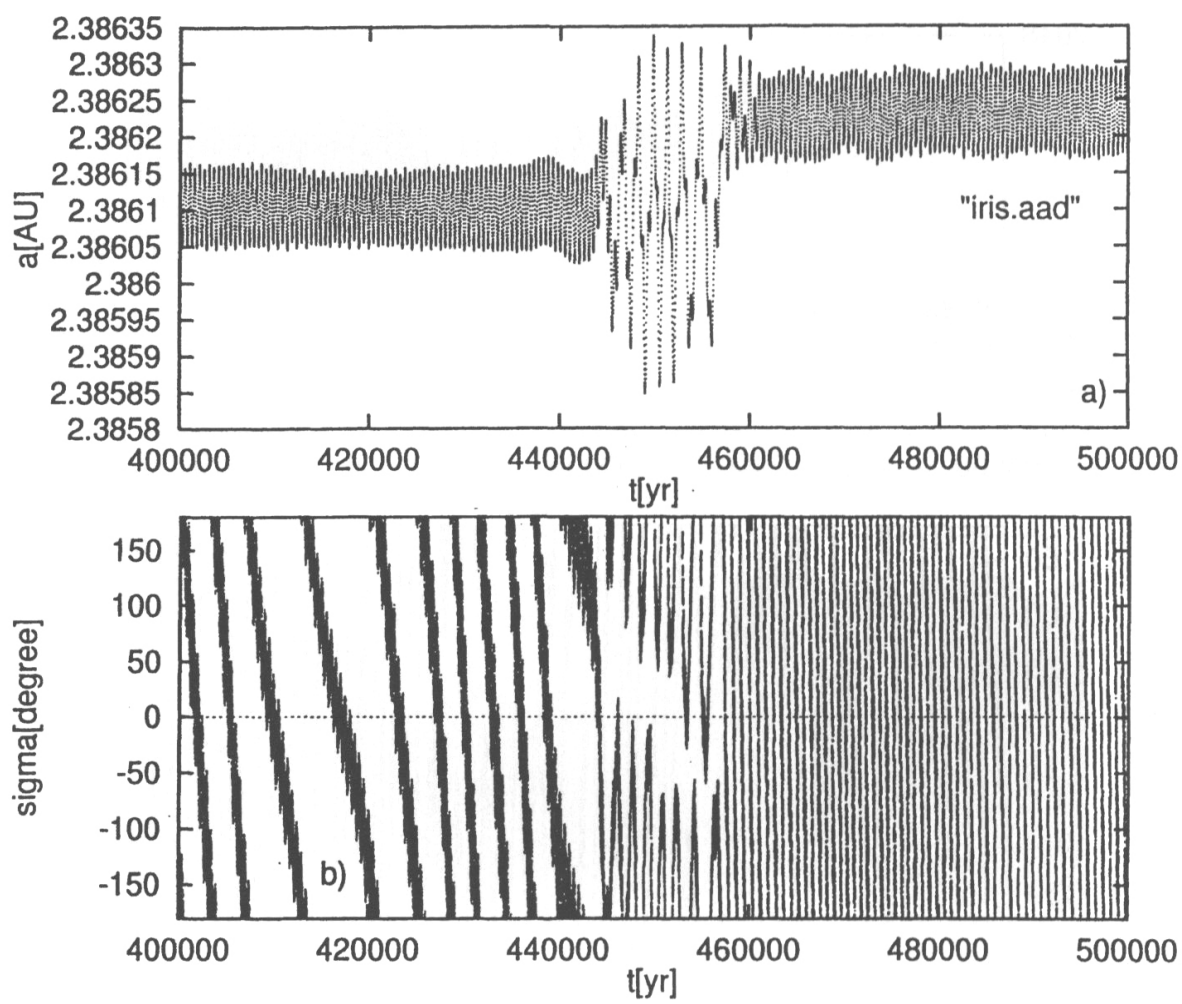

Fig. 4.

a) The filtered semimajor axis of Iris

b) The critical argument of Iris: $\sigma=49 \lambda-25 \lambda_{M}-24 \varpi$

$\sigma=49 \lambda-25 \lambda_{M}-24 \varpi$ for Iris. The resonances involved are of very high order and, as the sum of eccentricity powers in the resonant part of the perturbing function is equal to the order of the resonance, resonances of such high order were not suspected to be important. But it can be proved that the coefficients with the resonant terms are very high. For Flora, for instance, Nesvorný (1998) calculated the resonant term using his program in Mathematica:

$$
R_{\text {res }}=-1.3310^{10} \frac{M_{M}}{a_{M}} e_{M}^{7} e^{7} \cos \left(33 \lambda-19 \lambda_{M}-7 \varpi-7 \varpi_{M}\right)
$$

The discussion becomes even more complicated as the Laplacian expansion of the disturbing function (Mars-asteroid) may be divergent (Šidlichovský and Nesvorný, 1994).

Figures like Fig. 3 and Fig. 4 cannot be shown here for all the asteroids studied. For the asteroids of the first group (chaos coming from Mars) we found the 

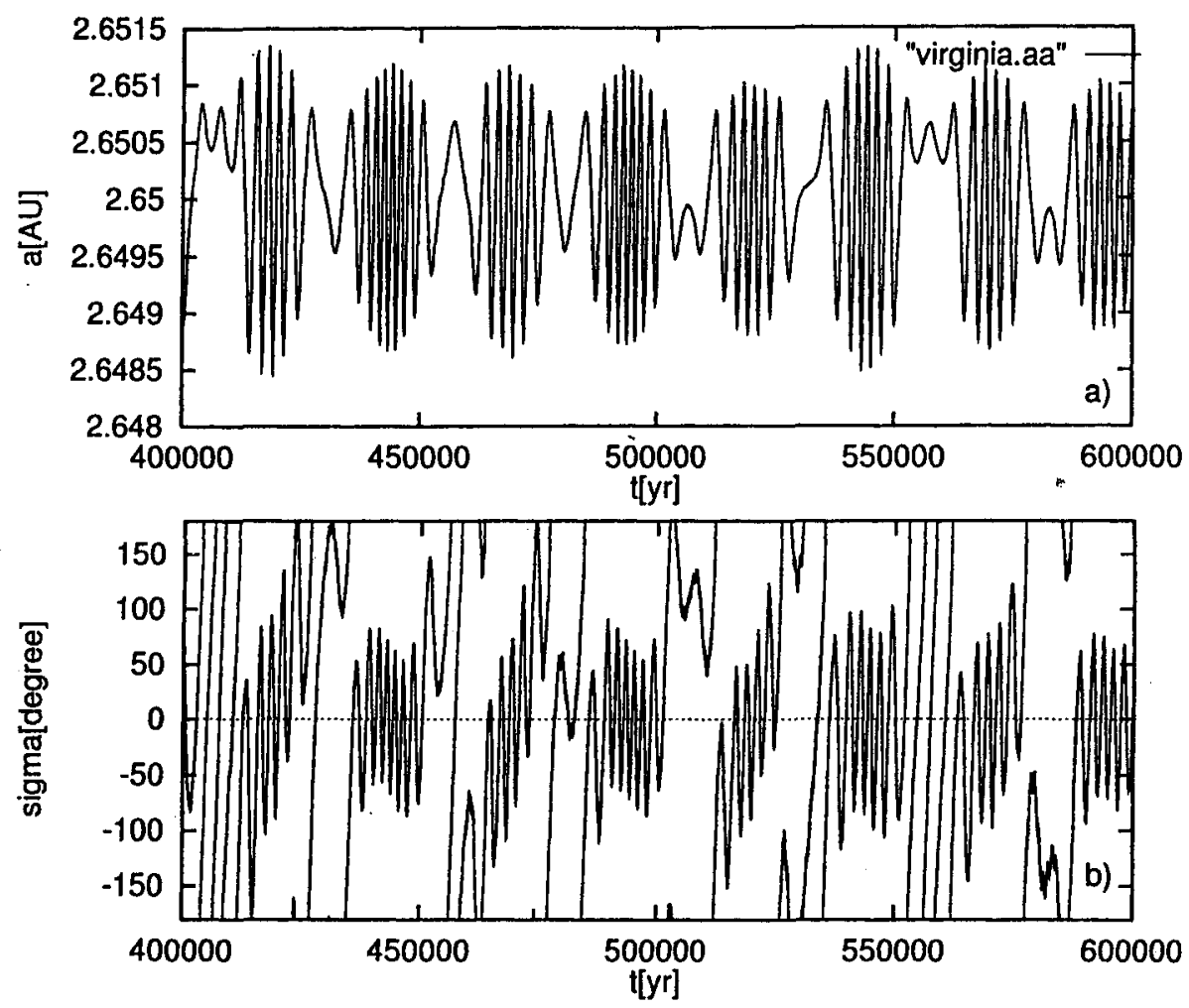

Fig. 5.

a) The filtered semimajor axis of Virginia

b) The critical argument of Virginia: $\sigma=11 \lambda_{J}-4 \lambda-\varpi_{J}-\varpi$

following resonances with Mars: Iris 25:49, Flora 19:33, Victoria 29:55, Eunomia 7:16, Daphne 9:22 while for Echo, Eurydike, Diana we did not succeed in finding the responsible resonance. The results are also shown in Column 5 of Tab. 1.

\section{Resonances with Jupiter}

For asteroids of the second group (strongest chaotic effects from the outer planets) we used the same method. Filtered $a$ suggests the candidates for the resonance, and the critical argument is the check. Figure 5 shows filtered $a$ and critical argument $\sigma=11 \lambda_{J}-4 \lambda-2 \varpi_{J}-5 \varpi$ for Virginia. We observed the following resonances with Jupiter: Pallas 18:7, Polyhymnia 22:9, Virginia 11:4, Semele 13:6. While for some of these asteroids, e.g., Virginia, the libration of $\sigma$, accompanied by a large variation of filtered $a$, is quite frequent, for some other, e.g., Pallas, only three such 

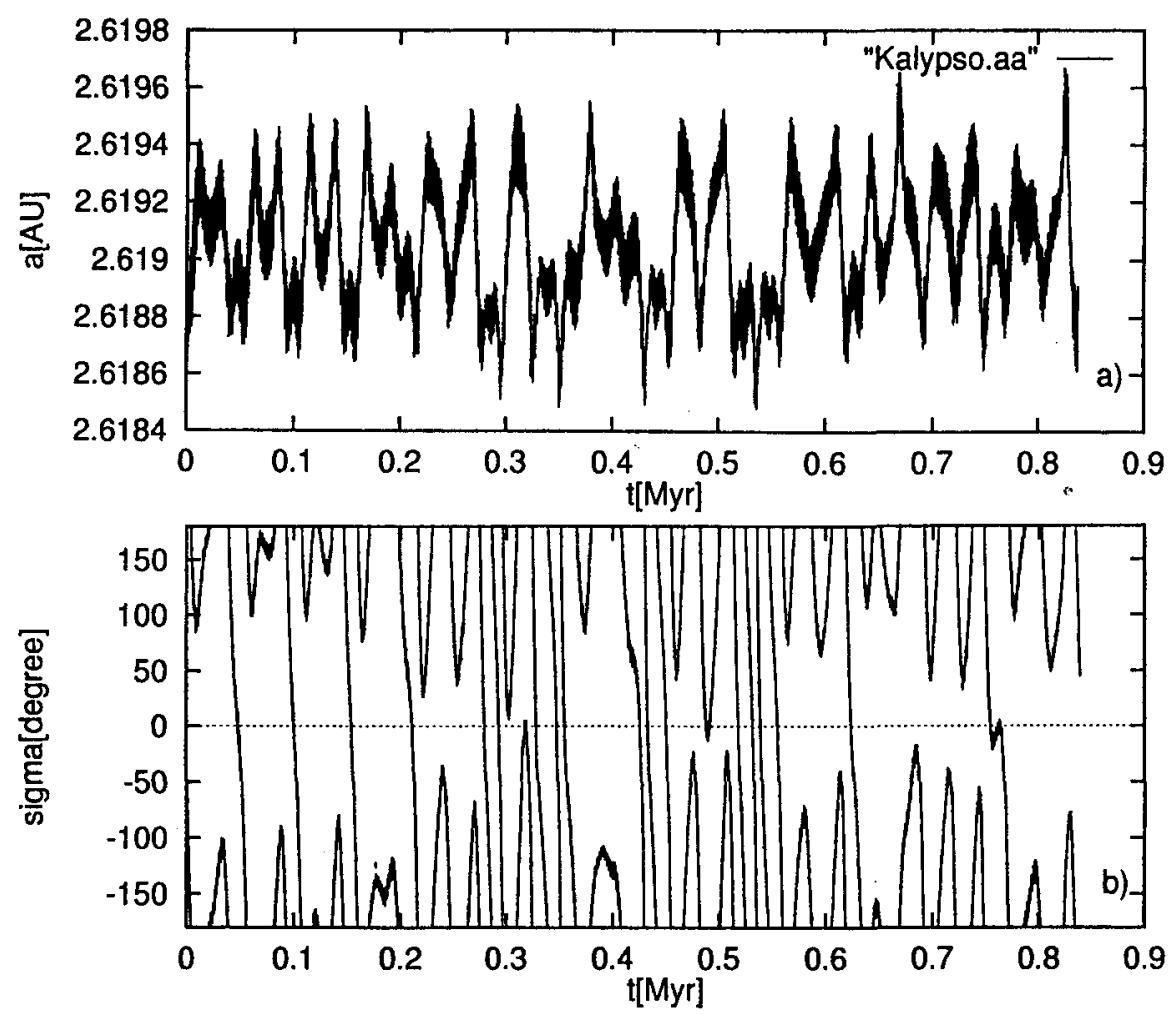

Fig. 6.

a) The filtered semimajor axis of Kalypso

b) The critical argument of Kalypso: $\sigma=6 \lambda_{J}-2 \lambda-\lambda_{S}-3 \varpi$

variations (in $800000 \mathrm{yrs}$ ) were found, each accompanied with just two or three libration cycles in $\sigma$.

\section{Three-Body Jupiter-Asteroid-Saturn Resonances}

We will denote the three-body resonance with the critical argument corresponding to Eq.(1) as the $m_{J}+m+m_{S}$ resonance. Resonance 6-2-1 has the critical argument $\sigma=6 \lambda_{J}-2 \lambda-\lambda_{S}$. Our list of resonances contained three-body resonances so that we could also check the three-body resonant arguments. Figure 6 shows filtered $a$ and critical argument $\sigma=6 \lambda_{J}-2 \lambda-\lambda_{S}-3 \varpi$ for Kalypso. We observed the following three-body resonances: Hygiea 8-3-4, Atlante 4-2+3, Kalypso 6-2-1, Panopea 2-1-2. 


\section{Conclusion}

For the 20 most chaotic asteroids among the first one hundred numbered asteroids, we studied the resonances with the strongest chaotic effect. The results are shown in the last column of Tab. 1. Many of the resonances are of a very high order, but calculations have shown that the coefficient standing with the resonant argument in the perturbing function can be unexpectedly large.

We checked more neighbouring resonances, but only one was always found important, at least for the period of time studied. We cannot, however, exclude the possibility of the effect of other high-order resonances later. For several subresonances of this resonance (the same coefficients with longitudes but different coefficients with perihelion longitudes in critical argument) we usually observed simultaneous libration which indicates overlap of these subresonances.

\section{Acknowledgements}

The support by Grant 205/98/1102 from the Grant Agency of the Czech Republic is gratefully acknowledged.

\section{References}

Froeschlé, Cl., Gonzi, R., Lega, E., Locatelli, U.: 1998, On the stochasticity of asteroid belt, it submitted to Celest. Mech. Dyn. Astron.

Holman ,M. J., Murray, N. W.: 1996, Chaos in high-order mean motion resonances in the outer asteroid belt, $A . J .112$, pp. 1278-1293

Laskar, J.: 1990, The Chaotic Motion in the Solar System. A Numerical Estimate of the Size of the Chaotic Zones, Icarus 88, pp. 266-291

Laskar, J., Froeschlé, Cl. and Celetti, A.: 1992, The Measure of Chaos by Numerical Analysis of the Fundamental Frequencies. Application to Standard Mapping, Physica D 56, pp. 253-269

Lega, E. and Froeschlé, Cl.: 1997, Fast Lyapunov Indicators Comparison with Other Chaos Indicators Application to Two and Four Dimensional Maps, in The Dynamical Behaviour of our Planetary System, Proceedings of the Fourth Alexandervon Humboldt Colloquium on Celestial Mechanics, editors: R. Dvorak, J. Henrard, Kluwer Academic Publishers, Dordrecht, pp. 257-275

Milani, A. and Nobili, A. M.: 1992, An example of stable chaos in the Solar System, Nature 357, pp. 569-571

Milani, A., Nobili, A.M. and Knežević,Z.: 1997, Stable Chaos in Asteroid Belt, Icarus, 125,pp. 13-31

Morbidelli, A. and Froeschlé, Cl: 1996, On the Relationship between Lyapunov Times and Macroscopic Instability Times, Celest. Mech. Dynam. Astron. 63, pp. 227-239

Morbidelli, A., Nesvorny, D.: 1998, Numerous weak resonances drive asteroids towards terrestrial planets orbits, submitted to Icarus

Nesvorný, D.: 1998, private communication

Nesvorný, D., Morbidelli, A.: 1998, An analytic model of three-body mean motion resonances, Celest. Mech. Dyn. Astron., in print

Quinlan, G.D. and Tremaine, S.: 1990, Symmetric Multistep Methods for the Numerical Integration of Planetary Orbits, A.J. 100, pp. 1694-1700

Quinn, T. R., Tremaine, S., Duncan, M.: 1991, A three million year integration of the Earth's orbit, A.J. 101, pp. 2287-2305

Šidlichovský, M., Nesvorný, D.: 1994, Temporary Capture of Grains in Exterior Resonances with the Earth,Astron. Astrophys. 289, pp. 972-982 
Šidlichovský, M. and Nesvorný, D.: 1997, Frequency Modified Fourier Transform and its Application to Asteroids, in The Dynamical Behaviour of our Planetary System, Proceedings of the Fourth Alexander von Humboldt Colloquium on Celestial Mechanics, editors: R. Dvorak, J. Henrard, Kluwer Academic Publishers, Dordrecht, pp. 137-148

Šidlichovsky, M. and Nesvorny, D.: 1998, A study of chaos in the asteroid belt, The Dynamics of Small Bodies in the Solar System: A Major Key to Solar System Studies, Proceedings of the NATO ASI in Maratea 1997, editors: A. E. Roy, B. A. Steves, Kluwer Academic Publishers, Dordrecht. 